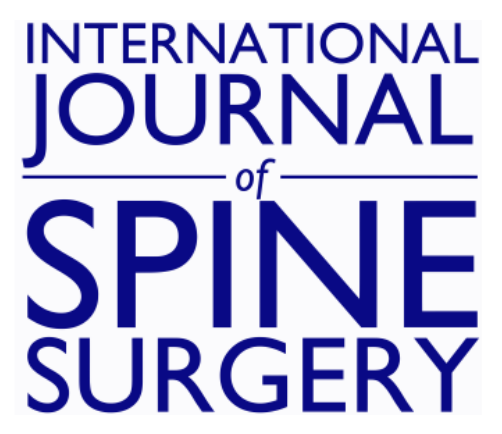

\title{
Anatomical Modifications during the Lateral Transpsoas Approach to the Lumbar Spine. The Impact of Vertebral Rotation.
}

ASHISH PATEL, JASON OH, DANTE LEVEN, FRANK S. CAUTELA, DIPAL CHATTERJEE, QAIS NAZIRI, FRANCESCO LANGELLA, BASSEL G. DIEBO and CARL B. PAULINO

Int J Spine Surg 2018, 12 (1) 8-14

doi: https://doi.org/10.14444/5002

http://ijssurgery.com/content/12/1/8

This information is current as of April 26, 2023.

Email Alerts Receive free email-alerts when new articles cite this article. Sign up at:

http://ijssurgery.com/alerts

The International Journal of Spine Surgery

2397 Waterbury Circle, Suite 1,

Aurora, IL 60504, Phone: +1-630-375-1432 


\title{
Anatomical Modifications during the Lateral Transpsoas Approach to the Lumbar Spine. The Impact of Vertebral Rotation.
}

\author{
ASHISH PATEL, MD,${ }^{1}$ JASON OH, MD,${ }^{1}$ DANTE LEVEN, DO,${ }^{1}$ FRANK S. CAUTELA, BS, ${ }^{1}$ DIPAL \\ CHATTERJEE, MD ${ }_{1}^{1}$ QAIS NAZIRI, MD, MBA, ${ }^{1}$ FRANCESCO LANGELLA, MD, ${ }^{2}$ BASSEL G. DIEBO, MD, ${ }^{1}$ \\ CARL B. PAULINO, MD ${ }^{1}$ \\ ${ }^{I}$ Department of Orthopaedic Surgery and Rehabilitation Medicine, SUNY Downstate Medical Center/University Hospital Brooklyn, Brooklyn, New York, \\ ${ }^{2}$ Orthopedic Division, Second University of Naples, Naples, Italy
}

\begin{abstract}
Background: We investigated impact of vertebral axial rotation on neurovascular anatomy in adult spinal deformity (ASD) patients and provided recommendations on the approach based on degree of axial rotation. In order to isolate vertebral rotation (VR) impact from the superimposed degenerative cascade observed in adulthood, adolescent idiopathic scoliosis (AIS) patients were analyzed.

Methods: Magnetic resonance imaging (MRI) scans (L1-S1) from 50 right-convex thoracic (left-convex lumbar) AIS patients were analyzed. At each intervertebral level, VR, lumbar plexus depth (LPD), and vascular structure depth (VSD) were evaluated. Paired $t$ test analyses were used to describe anatomic differences between the concave and convex aspect of our patients' curves. Correlation analysis was used to investigate relationships with soft tissue modifications and VR.

Results: Fifty AIS patients $(17 \mathrm{M}, 33 \mathrm{~F})$ with mean thoracic Cobb of $50.6^{\circ} \pm 17.0^{\circ}$ and mean lumbar Cobb of $41.9^{\circ}$ $\pm 13.0^{\circ}$ were included. Mean VR at each level was $\mathrm{L} 1-2=-6.6^{\circ}, \mathrm{L} 2-3=-7.7^{\circ}, \mathrm{L} 3-4=-6.5^{\circ}, \mathrm{L} 4-5=-4.7^{\circ}, \mathrm{L} 5-\mathrm{S} 1=-2.6^{\circ}$ (negative value denotes clockwise rotation). We found significant differences $(P<.05)$ between concave-convex (rightleft) LPD at each level (L1-2 $=3.7 \mathrm{~mm}, \mathrm{~L} 2-3=5.1 \mathrm{~mm}, \mathrm{~L} 3-4=4.2 \mathrm{~mm}, \mathrm{~L} 4-5=2.2 \mathrm{~mm}, \mathrm{~L} 5-\mathrm{S} 1=2.2 \mathrm{~mm}$ ). Vascular structure depth was significantly different at L1-L2 $(3.2 \mathrm{~mm})$ and L5-S1 $(3 \mathrm{~mm})$. Significant correlation was found between increasing VR and concave-convex LPD difference $(r=0.68, P<.001)$.

Conclusions: This study demonstrates that displacement of the lumbar plexus is tied to the magnitude of VR in patients with AIS. When approaching the lumbar spine, this displacement widens the safe surgical corridor on the convex side and narrows the corridor on the concave side.

Level of Evidence: IV

Clinical Relevance: Preoperative review of MRI scans should occur to assess the patient's safe surgical corridor for lateral lumbar interbody fusion (LLIF). Adult spinal deformity surgeons who approach a degenerated spine in patients with progressive AIS in adulthood must carefully plan for patient positioning, neurovascular anatomy, and realignment objectives prior to the day of surgical intervention.
\end{abstract}

New Technology

Keywords: vertebral rotation, lateral lumbar interbody fusion, LLIF, patient specific approach, precision medicine

\section{INTRODUCTION}

The increase in the prevalence of adult spinal deformity (ASD) in recent years is highly concerning. ${ }^{1}$ Adult spinal deformity exhibits a variety of clinical presentations, including de novo degenerative scoliosis (DS), adolescent idiopathic scoliosis in the adult (AAIS), iatrogenic sagittal deformity, and secondary DS due to musculoskeletal or systemic diseases. When approaching ASD patients, there are no special considerations for DS versus AAIS in preoperative planning. In fact, a recent study by Guler et al. and the European Spine Study Group ${ }^{2}$ revealed that, in the absence of sagittal malalignment, surgeons might misdiagnose DS for AAIS when looking at the radiographs. Overall, ASD affects a heterogeneous population of patients that might require different treatment or surgical considerations based on etiology.,

Minimally invasive surgery, most notably lateral lumbar interbody fusion (LLIF), is being increasingly recognized as a surgical option for spinal 
deformity patients. ${ }^{5,6}$ An increasingly utilized approach includes multilevel interbody devices using the LLIF approach, followed by posterior osteotomies and instrumentation, for coronal and sagittal deformity correction in the lumbar spine. While LLIF carries numerous advantages, ${ }^{7,8}$ there are reports of vascular injuries as well as sensory and motor neurological injuries. ${ }^{9-11}$ This is either due to direct injury or retractor-related compression of the nerve roots posteriorly and the vessels anteriorly. Anatomical studies related to LLIF safety have thus far been limited to subjects free of rotational deformity. ${ }^{12}$ To date, the literature does not mention the assessment and consideration of axial plane deformity when using the LLIF technique. In fact, AAIS and DS patients are approached in a similar surgical and anatomical manner, ${ }^{13,14}$ when perhaps certain differences should be considered.

In the era of personalized medicine, there is an increased need for a more patient-specific approach to managing the spine. While there is extensive knowledge regarding the bony alignment of the spine in both asymptomatic and deformed patients, the literature lacks analysis of soft tissues, such as muscle and neurovascular structures. In this study, we aimed to investigate the impact of vertebral axial rotation (VAR) on the neurovascular anatomy in patients with spinal deformity. Adolescent idiopathic scoliosis (AIS) patients were selected to isolate vertebral rotation (VR) analysis without confounding the findings with typical superimposed degenerative cascade observed in adulthood. The authors hypothesized that lumbar VR progressively alters the anatomy of the adjacent soft tissues. With the data gathered, we also aimed to provide recommendations on the approach based on the degree of axial rotation observed.

\section{MATERIALS AND METHODS}

\section{Study Design}

This is a retrospective review of a prospectively collected database. After International Review Board (IRB) approval, patients were consecutively enrolled at a single center between 2007 and 2013. The criteria for inclusion were AIS patients with right convex thoracic curve (Cobb angle $>50^{\circ}$, with compensatory/structural left convex lumbar curve), availability of magnetic resonance imaging (MRI) of the lumbar spine between L1 and S1 vertebrae, and 36-inch full-spine coronal and sagittal radio- graphs. Patients were surgical candidates that underwent MRI to rule out spinal cord pathology prior to surgical intervention. The criteria for exclusion were patients with abnormal neurological findings on physical exam or MRI, history of previous surgery, and/or poor resolution MRI precluding measurement of the lumbar plexus. The senior author is a paid presenter for DePuy, a Johnson \& Johnson company. The remaining authors report no conflict of interest concerning the materials or methods used in this study or the findings specified in this paper. This study was performed without external funding.

\section{Data Collection}

Demographic data included patients' age and gender. Radiographs were measured using a dedicated, validated software. ${ }^{15}$ A single 1.5 Tesla MRI scanner was used to acquire continuous axial T2 weighted images for all patients. Images were appropriately angled to the disc spaces. Two readers, a chief orthopaedic resident and a senior musculoskeletal radiologist, independently analyzed MRI scans using a picture archiving and communication system. The methodology of the measurement is reliable based on the literature. ${ }^{12}$ The intraclass correlation coefficients for interobserver and intraobserver reliabilities were reported as 0.74 and 0.87 , respectively. ${ }^{12}$

\section{Magnetic Resonance Imaging Parameters}

At each vertebral level, the anterior vertebral body (AVB) line was defined as a horizontal line tangential to the most anterior aspect of the vertebral disc. Plumb lines were measured from the AVB to each of the anatomical structures of interest. Vascular structure depth (VSD) was defined as the distance in millimeters from the AVB to the most posterior edge of the great vessels. Lumbar plexus depth (LPD) was defined as the distance in millimeters from the AVB to the most anterior fascicle (branch) of the lumbar plexus (Figure 1).

In a secondary analysis, the same parameters were reevaluated using a methodology that controlled for the VAR. In this method, corrected AVB (CAVB) line was considered as the perpendicular to the vertebral bisector (VB: a line bisects the vertebral disc, spinal canal, and posterior elements into symmetric halves). The VR was defined as the angle between the AVB and the CAVB; it is also defined in the literature as RAsag by Aaro and 


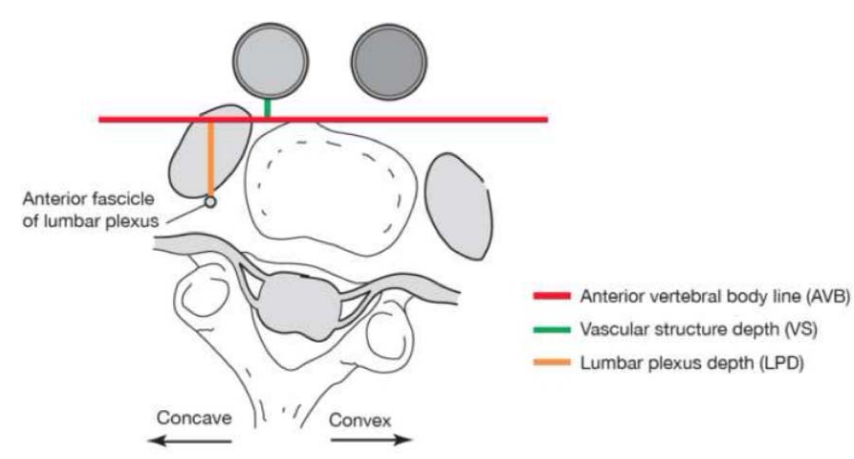

Figure 1. Illustration of magnetic resonance imaging parameters using direct measurement of the most anterior aspect of the vertebral disc.

Dahlborn. ${ }^{16}$ Theoretically, measurements from this secondary analysis should more closely resemble intraoperative findings since a true lateral approach to the vertebral disc is desired when using the LLIF technique (Figures 2 and 3).

\section{Statistical Methods}

Descriptive analyses included means and standard deviations for all demographic, radiographic, and MRI parameters. The Shapiro-Wilk test was used to assess the normality of the data. Lumbar plexus and VSDs were compared between lumbar curve convexity (left) and concavity (right) using student $t$ test statistical analysis. Pearson correlation was used to analyze linear relationships between spinal deformity parameters and differences in LPD and VS between right and left sides. The same analyses were performed using measurements obtained from the methodology that corrected for VAR. Statistical analysis was performed using IBM SPSS Statistics v. 20.0.0 (SPSS Inc., 2009, Chicago, IL). The level of significance was set at $P<.05$.

\section{RESULTS}

\section{Patient Population}

Fifty patients met the inclusion/exclusion criteria. The patient sample had a mean age of $18.4 \pm 8.9$ years old, and 33/50 (66\%) were females. Mean thoracic coronal Cobb was $50.6^{\circ} \pm 17.0^{\circ}$ and mean lumbar coronal Cobb was $41.9^{\circ} \pm 13.0^{\circ}$. Maximum VR was reported at L2-L3 $\left(-7.69^{\circ} \pm 7.8^{\circ}\right)$; the mean VRs at other levels are listed in Table 1.

\section{Direct (Uncorrected) Analysis}

Vascular structure depth was significantly more posterior in the right side (concave side) at the levels of L1-2 and L5-S1 (Table 2). Lumbar plexus depth

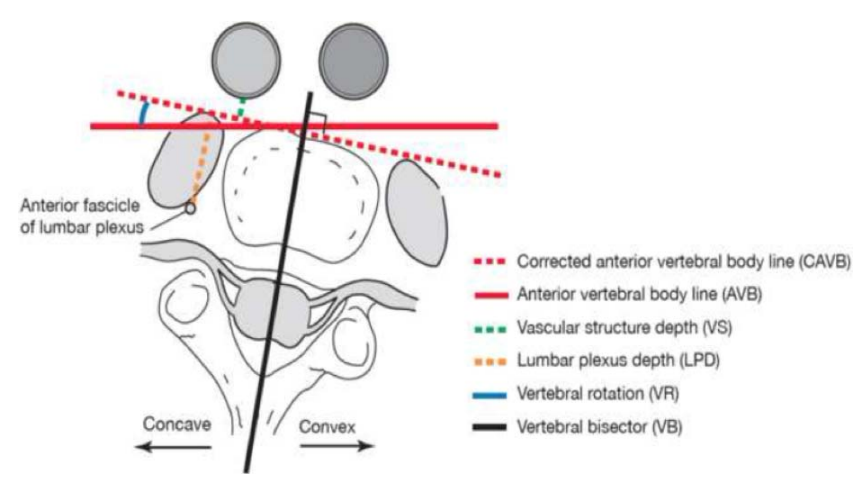

Figure 2. Illustration of magnetic resonance imaging parameters after correcting for axial rotation by considering anterior vertebral body line as the tangent to the vertebral bisector.

was significantly different between the convex and concave side at each vertebral level. Specifically, at the level of L2-3, the mean difference of LPD was $6.1 \mathrm{~mm}$, with the right (concave) side being anterior, ie, closer to the surgical corridor (Table 2).

Safe surgical corridors in convex and concave sides are illustrated in Figure 4.

\section{Corrected Axial Rotation Analysis (True Lateral Approach)}

Vascular structure depth was significantly more posterior in the right side (concave side) at the levels of L1-2, L2-3, and L4-5 (Table 3). Similarly, LPD was significantly different between the convex and concave sides at the levels of L1-2 and L4-5 (Table 3).

Safe surgical corridors in convex and concave sides after correction of axial rotation are illustrated in Figure 5.

Pearson correlation revealed a significant positive correlation between magnitude of VR and difference in LPD between convex and concave sides $(r=$ $0.680, P<.001)$, but not with difference in VSD.

\section{DISCUSSION}

This study demonstrates that displacement of the lumbar plexus is tied to the magnitude of $\mathrm{VR}$ in patients with AIS. When approaching the lumbar

Table 1. Mean and standard deviations of vertebral rotation at each lumbar level. Negative value denotes clockwise rotation.

\begin{tabular}{lc}
\hline Vertebral Level & Mean Axial Rotation \\
\hline L1-2 & $-6.6^{\circ} \pm 9.7^{\circ}$ \\
L2-3 & $-7.7^{\circ} \pm 7.8^{\circ}$ \\
L3-4 & $-6.5^{\circ} \pm 6.3^{\circ}$ \\
L4-5 & $-4.7^{\circ} \pm 5.1^{\circ}$ \\
L5-S1 & $-2.6^{\circ} \pm 4.8^{\circ}$ \\
\hline
\end{tabular}




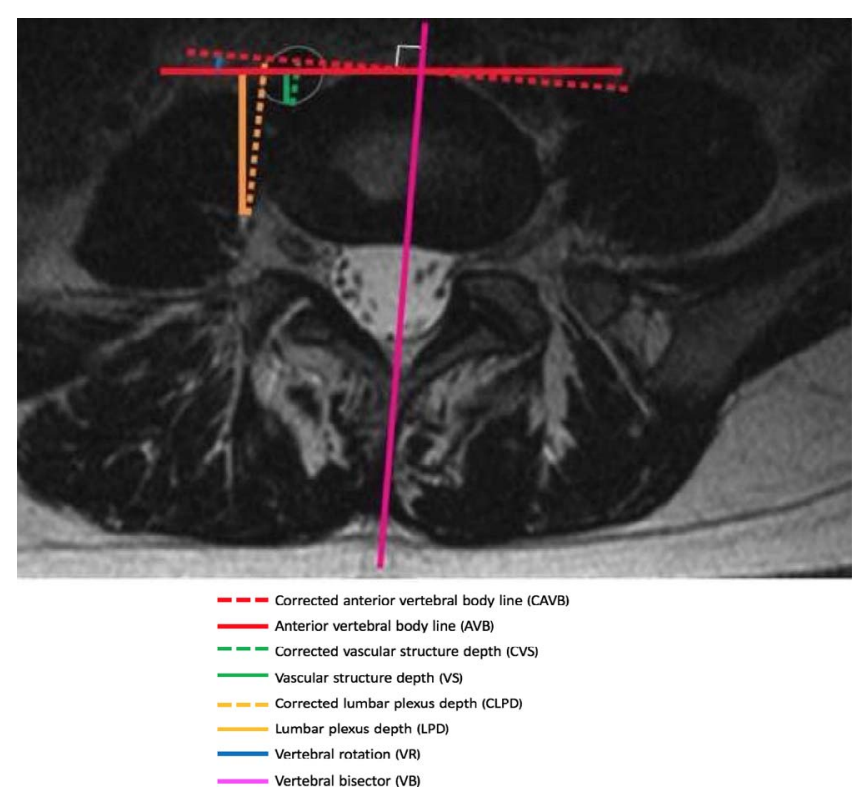

Figure 3. Axial magnetic resonance imaging displaying parameters using direct measurement of the most anterior aspect of the vertebral disc and after correcting for axial rotation by considering anterior vertebral body line as the tangent to the vertebral bisector.

spine in this patient group, this displacement widens the safe surgical corridor on the convex side and narrows the corridor on the concave side. After controlling for the VAR via a true lateral approach to the vertebral level, the data revealed lessening of these left- and right-sided differences. Interestingly, the anatomy of the great vessels was less influenced by the vertebral body rotation than the psoas/ lumbar plexus anatomy. However, the data showed that, when controlling for axial rotation with a true lateral approach, the vascular structures are encountered within the surgical corridor on the concave side, reducing the safe passage to the vertebral disc and potentially increasing the likelihood for direct or retractor based vascular injury. More importantly, the maximum displacement was at the level of the maximum VR (L2-3, $11.9 \mathrm{~mm}$ within the anterior vertebral line).

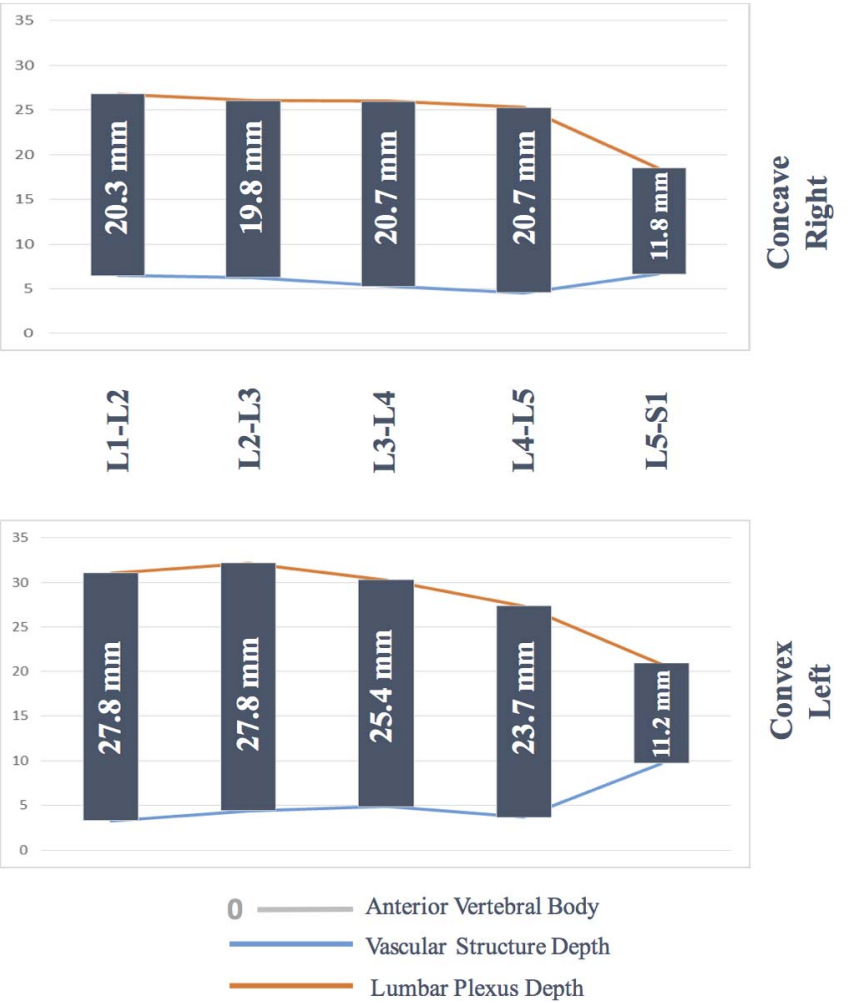

Figure 4. Safe surgical corridors in convex and concave side of the lumbar curve by level. In the literature, mean superior and inferior widths of the lumbar vertebrae are reported between $33.5-34.9 \mathrm{~mm}$ by Gilad and Nissan. ${ }^{15}$

To our knowledge, this is the first study to isolate and evaluate the impact of axial rotational deformity on the position of neurovascular structures that may be endangered when performing the LLIF approach. More rotation was associated with greater disparity of the surgical safe corridors between the curve concavity and the curve convexity. Specifically, our data indicate that, for patients with vertebral body rotation, the safe surgical corridor for the transpsoas approach increases when approaching from the curve convexity (Figure 6).

The literature suggests that the lateral approach from the concave side provides more comfortable

Table 2. Details of the vascular structure and lumbar plexus depth differences between convex and concave sides at each vertebral level of the lumbar spine. Of note, vascular structures are anterior to the vertebral body, and more depth means displacing posteriorly to the surgical corridor, whereas lumbar plexuses are posterior to the vertebral body, and more depth means displacing further posteriorly from the surgical corridor.

\begin{tabular}{|c|c|c|c|c|c|c|}
\hline \multirow[b]{2}{*}{ Lumbar Level } & \multicolumn{3}{|c|}{ Vascular Structure Depth, mm } & \multicolumn{3}{|c|}{ Lumbar Plexus Depth, mm } \\
\hline & Left (Convex) & Right (Concave) & $P$ Value & Left (Convex) & Right (Concave) & $P$ Value \\
\hline L1-2 & 3.3 & 6.5 & $<.001$ & 31.1 & 26.8 & $<.001$ \\
\hline L2-3 & 4.4 & 6.3 & .36 & 32.2 & 26.1 & $<.001$ \\
\hline L3-4 & 4.9 & 5.3 & .39 & 30.3 & 26 & $<.001$ \\
\hline L4-5 & 3.7 & 4.6 & .09 & 27.4 & 25.3 & .01 \\
\hline L5-S1 & 9.7 & 6.7 & $<.001$ & 20.9 & 18.5 & $<.001$ \\
\hline
\end{tabular}




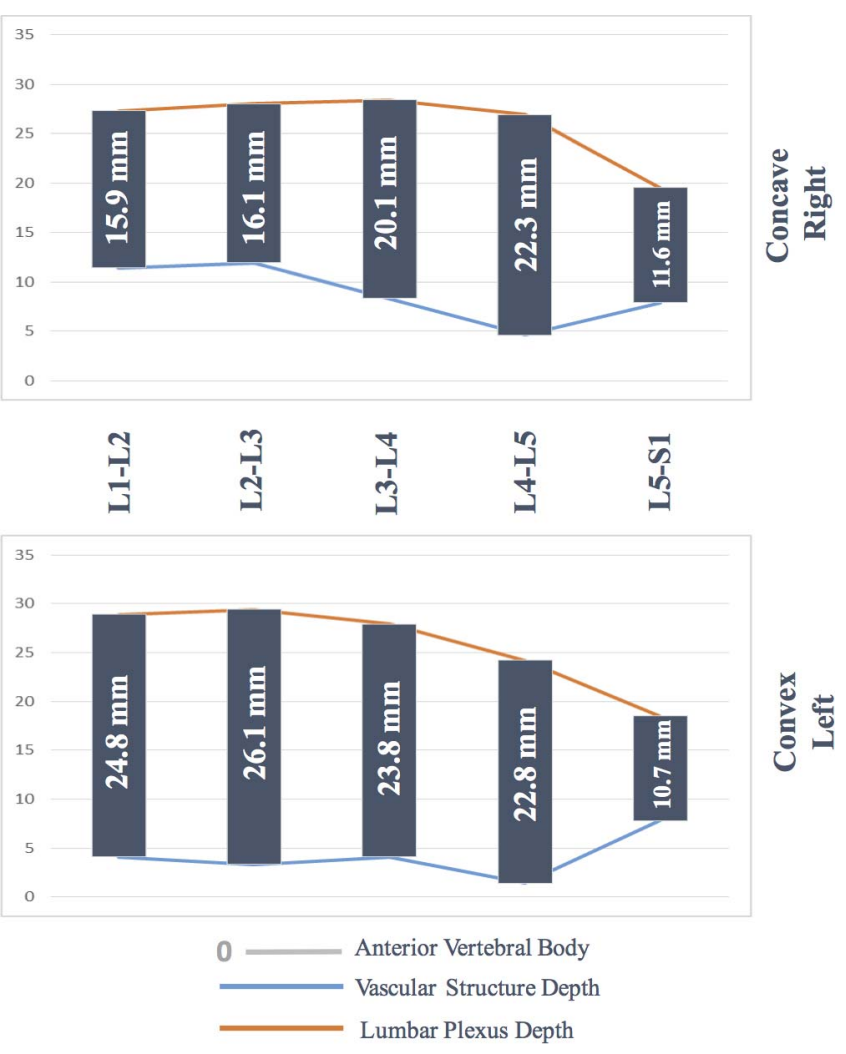

Figure 5. Safe surgical corridor in convex and concave side of the lumbar curve by level following controlling for axial rotation. In the literature, mean superior and inferior widths of the lumbar vertebrae are reported between 33.5$34.9 \mathrm{~mm}$ by Gilad and Nissan. ${ }^{17}$

access to several disc levels with minimal number of incisions. In addition, this side carries less risk of endplate violation, better annulus release, and interbody placement. ${ }^{8}$ However, advantages to utilizing the transpsoas approach from the curve convexity include improved proximity of the spine to the surgeon (as compared to access on the curve concavity) and favorable wedging of the vertebral disc. ${ }^{17}$ Several studies found that there was a greater risk of neurovascular damage when operating on patients' right-concave side as compared to patients' left. ${ }^{12}$ Similarly, Anand et al. stressed their preference for the convex side when performing LLIF. ${ }^{18}$ The variation of these reports highlights the importance of our study and supports the idea that axial anatomy is a significant player in determining which side of the deformity is safer for surgical access. We recommend careful review of preoperative computed tomography (CT)/MRI scans prior to surgery for assessing any potential patient-specific anatomic variations, especially in the patients with progressed AIS in adulthood.

The surgical safe zone has historically been described as the anterior half of the vertebral disc space in a true lateral projection. In a normal spine, the lumbar plexus courses from the posterior edge of the vertebral disc in the proximal lumbar spine to the junction of the posterior middle-third of the disc at L4-5. ${ }^{19}$ The great vessels remain anterior to the vertebral body in the upper lumbar spine in most cases. Moving distally, the vessels may displace posteriorly to the anterior $25 \%$ of the vertebral space. ${ }^{20}$ However, one size does not fit all. For the first time, this study provided level specific surgical safe corridor in each side of the lumbar curve (Figures 4 and 5). Data on L5-S1 was included for completeness sake, but it should be noted that this is not a surgically accessible level for LLIF.

The lateral transpsoas approach to the lumbar spine is a useful tool in the spine surgeon's arsenal; however, it is not acceptable to perform LLIF without addressing patient specific deformity, mainly the VAR. Different rotations lead to different anatomy of the soft tissue surrounding the lumbar spine. In clinical practice, each patient's fine-cut MRI scans should be carefully reviewed preoperatively to assess their individual safe surgical corridor for the LLIF approach. When AIS patients present with spinal deformity in adulthood, degenerative cascade such as disc wedging and olisthesis has already complicated the clinical and radiographic presentation. To avoid these confounding factors, the authors investigated the

Table 3. Comparison between vascular structure and lumbar plexus depth between convex and concave sides of the lumbar curve after controlling for axial plane rotation. Of note, vascular structures are anterior to the vertebral body, and more depth means displacing posteriorly to the surgical corridor, whereas lumbar plexuses are posterior to the vertebral body, and more depth means displacing further posteriorly from the surgical corridor.

\begin{tabular}{|c|c|c|c|c|c|c|}
\hline \multirow[b]{2}{*}{ Lumbar Level } & \multicolumn{3}{|c|}{ Vascular Structure Depth, mm } & \multicolumn{3}{|c|}{ Lumbar Plexus Depth, mm } \\
\hline & Left (Convex) & Right (Concave) & $P$ Value & Left (Convex) & Right (Concave) & $P$ Value \\
\hline L1-2 & 4.1 & 11.4 & $<.001$ & 28.9 & 27.3 & $<.001$ \\
\hline L2-3 & 3.3 & 11.9 & $<.001$ & 29.4 & 28 & .38 \\
\hline L3-4 & 4.1 & 8.3 & .10 & 27.9 & 28.4 & .28 \\
\hline L4-5 & 1.4 & 4.6 & $<.001$ & 24.2 & 26.9 & $<.001$ \\
\hline L5-S1 & 7.8 & 7.9 & .48 & 18.5 & 19.5 & .16 \\
\hline
\end{tabular}



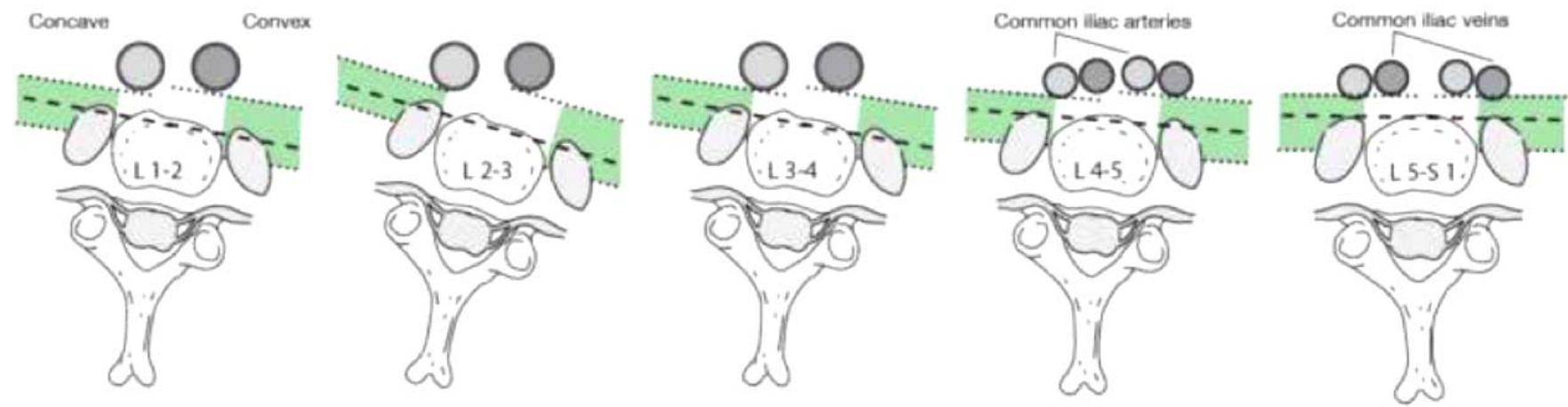

Figure 6. Influence of vertebral rotation on convex versus concave safe surgical corridor for lateral lumbar interbody fusion.

impact of spinal rotation on lumbar soft tissue anatomy in adolescence. Adult spinal deformity surgeons who approach a degenerated spine in patients with AIS in adulthood must carefully plan for patient positioning, neurovascular anatomy, and realignment objectives prior to the day of surgical intervention.

\section{Limitations}

The authors appreciate that this study had several limitations. First, data concerning patient race and ethnicity were not recorded. Second, the study was based entirely on imaging data without clinical correlation. The use of AIS patients rather than ASD patients is another limitation of this study. This may limit the ability to extrapolate these results to a clinical setting involving ASD patients. Another limitation that should be noted is the fact that only AIS patients with main thoracic curves were analyzed in this study. In clinical practice, the best indication for LLIF is those patients with main lumbar or thoracolumbar curves. An area of future research can involve studying the lateral transpsoas approach in patients with these curves. In addition, investigating the impact of using our corridor on the surgical outcomes of LLIF is of utmost importance. Nevertheless, this study is the first step in investigating modifications of the paraspinal soft tissues in patients with spinal deformity. Further studies are needed to investigate whether the rotatory subluxation in DS has the same impact on the neurovascular anatomy of the psoas/lumbar complex. ${ }^{21}$

\section{CONCLUSION}

The lateral transpsoas approach to the lumbar spine is an increasingly popular minimally invasive technique for treatment of spinal pathologies, including spinal deformity. Our study indicates that there is an anatomic advantage to approaching the spine from the side of the curve convexity as opposed to the curve concavity. Moreover, we demonstrated that preoperative planning for LLIF should be patient specific. Specifically, the VAR at the level of interest should be assessed for possible modification of the surrounding soft tissue anatomy.

\section{REFERENCES}

1. Schwab FJ, Dubey A, Gamez L, et al. Adult scoliosis: prevalence, SF-36, and nutritional parameters in an elderly volunteer population. Spine (Phila Pa 1976). 2005;30(9):10821085.

2. Guler UO, Yuksel S, Yakici S, et al.; European Spine Study Group. Analysis of the reliability of surgeons' ability to differentiate between idiopathic and degenerative spinal deformity in adults radiologically. What descriptive parameters help them decide? Eur Spine J. 2016;25(8):2401-2407. https://doi. org/10.1007/s00586-015-4366-3

3. Berjano P, Lamartina C. Classification of degenerative segment disease in adults with deformity of the lumbar or thoracolumbar spine. Eur Spine J. 2014;23(9):1815-1824. https://doi.org/10.1007/s00586-014-3219-9

4. Acaroğlu RE, Dede Ö, Pellisé F, et al.; European Spine Study Group. Adult spinal deformity: a very heterogeneous population of patients with different needs. Acta Orthop Traumatol Turc. 2016;50(1):57-62. https://doi.org/10.3944/ AOTT.2016.14.0421

5. Eck JC, Hodges S, Humphreys SC. Minimally invasive lumbar spinal fusion. J Am Acad Orthop Surg. 2007;15(6):321329.

6. Park P, Wang MY, Lafage V, et al.; International Spine Study Group. Comparison of two minimally invasive surgery strategies to treat adult spinal deformity. J Neurosurg Spine. 2015;22(4):374-380. https://doi.org/10.3171/2014.9.SPINE131004

7. Dakwar E, Cardona RF, Smith DA, Uribe JS. Early outcomes and safety of the minimally invasive, lateral retroperitoneal transpsoas approach for adult degenerative scoliosis. Neurosurg Focus. 2010;28(3):E8. https://doi.org/10. 3171/2010.1.FOCUS09282

8. Berjano P, Lamartina C. Far lateral approaches (XLIF) 
in adult scoliosis. Eur Spine J. 2013;22(Suppl 2):242-253. https://doi.org/10.1007/s00856-012-2426-5

9. Yuan PS, Rowshan K, Verma RB, Miller LE, Block JE. Minimally invasive lateral lumbar interbody fusion with direct psoas visualization. J Orthop Surg Res. 2014;9(1):20. https:// doi.org/10.1186/1749-799X-9-20

10. Cahill KS, Martinez JL, Wang MY, Vanni S, Levi AD. Motor nerve injuries following the minimally invasive lateral transpsoas approach. J Neurosurg Spine. 2012;17(3):227-231. https://doi.org/10.3171/2012.5.SPINE1288

11. Joseph JR, Smith BW, La Marca F, Park P. Comparison of complication rates of minimally invasive transforaminal lumbar interbody fusion and lateral lumbar interbody fusion: a systematic review of the literature. Neurosurg Focus. 2015;39(4):E4. https://doi.org/10.3171/2015.7.FOCUS15278

12. Kepler CK, Bogner EA, Herzog RJ, Huang RC. Anatomy of the psoas muscle and lumbar plexus with respect to the surgical approach for lateral transpsoas interbody fusion. Eur Spine J. 2011;20(4):550-556. https://doi.org/10.1007/ s00586-010-1593-5

13. Than KD, Park $\mathrm{P}, \mathrm{Fu} \mathrm{K}$, et al. Clinical and radiographic parameters associated with best versus worst clinical outcomes in minimally invasive spinal deformity surgery. J Neurosurg Spine. 2016; 25(1):21-25. https://doi.org/ 10.3171/2015.12.SPINE15999

14. Park P, Okonkwo DO, Nguyen S, et al.; International Spine Study Group. Can a minimal clinically important difference be achieved in elderly patients with adult spinal deformity who undergo minimally invasive spinal surgery? World Neurosurgery. 2016;86:168-172.

15. Lafage R, Ferrero E, Henry JK, et al. Validation of a new computer-assisted tool to measure spino-pelvic parameters. Spine J. 2015;15(12):2493-2502. https://doi.org/10.1016/j.spinee. 2015.08.067

16. Aaro S, Dahlborn M. The longitudinal axis rotation of the apical vertebra, the vertebral, spinal, and rib cage deformity in idiopathic scoliosis studied by computer tomography. Spine (Phila Pa 1976). 1981;6(6):567-572. https://doi.org/10.1097/ 00007632-198111000-00007

17. Gilad I, Nissan M. Sagittal evaluation of elemental geometrical dimensions of human vertebrae. $J$ Anat. 1985;143:115-120.

18. Anand N, Baron EM, Khandehroo B, Kahwaty S. Long-term 2- to 5-year clinical and functional outcomes of minimally invasive surgery for adult scoliosis. Spine (Phila Pa
1976). 2013;38(18):1566-1575. https://doi.org/10.1097/BRS. 0b013e31829cb67a

19. Spivak JM, Paulino CB, Patel A, Shanti N, Pathare N. Safe zone for retractor placement to the lumbar spine via the transpsoas approach. 2013;21(1):77-81.

20. $\mathrm{Hu} \mathrm{H}$, Meijer OG, van Dieën JH, et al. Is the psoas a hip flexor in the active straight leg raise? Eur Spine $J$. 2011;20(5):759-765. https://doi.org/10.1007/s00586-010-1508-5

21. Ferrero E, Lafage R, Challier V, et al. Clinical and stereoradiographic analysis of adult spinal deformity with and without rotatory subluxation. Orthop Traumatol Surg Res. 2015;101(5):613-618. https://doi.org/10.1016/j.otsr.2015.04.008

Disclosures and COl: Carl B. Paulino, MD, is a paid presenter for DePuy, a Johnson \& Johnson company and Ethicon, a Johnson \& Johnson company. The remaining authors report no conflict of interest concerning the materials or methods used in this study or the findings specified in this paper. This study was performed without external funding.

IRB Approval: This study was approved by the SUNY Downstate Medical Center Institutional Review Board (study \# 321384-1).

Corresponding Author: Bassel G. Diebo, MD, Department of Orthopaedic Surgery and Rehabilitation Medicine, SUNY Downstate Medical Center/University Hospital Brooklyn, 450 Clarkson Avenue, Box 30, Brooklyn, NY 11203. Mobile: (260) 797-1345; Phone: (718) 221-5270; Email:dr.basseldiebo@gmail.com.

Published 30 March 2018

This manuscript is generously published free of charge by ISASS, the International Society for the Advancement of Spine Surgery. Copyright (C) 2018 ISASS. To see more or order reprints or permissions, see http://ijssurgery.com. 